\section{A Simple Tracking Control for Chua's Circuit}

Hector Puebla, Jose Alvarez-Ramirez, and Ilse Cervantes

\begin{abstract}
This brief addresses the problem of designing a robust tracking controller for a nonlinear Chua's circuit involving plant uncertainties and external disturbances. Given a bounded desired trajectory, a robust tracking controller, which is based on modeling-error compensation is developed such that all the states and signals of the closed-loop system are bounded and the tracking error can be made as small as desired. In contrast to the previous proposed controllers for tracking control of Chua's circuit, the controller developed here is linear and equivalent to a traditional PI compensator.
\end{abstract}

Index Terms-Chua's circuit, modeling-error compensation, tracking.

\section{INTRODUCTION}

In the last decade, Chua's circuits have became a standard prototype to study the dynamics and control of nonlinear circuits. The reason is that Chua's circuit is a simple system that exhibits a wide variety of nonlinear dynamics phenomena such as bifurcations, chaos, and uncertain functions and parameters. The Chua's circuit (chaotic) dynamics and bifurcations have been widely studied and numerous results can be found in the literature (see [1]). Regarding the operation of Chua's circuits, a significant research attention has been paid on studying the control problem. Fuzzy [2], [3], time-series-based [4] linear matrix inequalities [5] and digital-redesign [6] methodologies are a few examples of control-design approaches used for controlling Chua's circuits. Numerical simulations and experimental experiences based on digital control implementation have demonstrated the performance of the reported controllers. A major drawback of these control-design approaches is that they lead to controllers with a complex structure. For instance, the implementation of adaptive fuzzy control approaches [2], [3] requires that the circuitry realization of a nonlinear controller which, in most cases, is more complex than Chua's circuit. In practice, controllers are commonly implemented with inexpensive simple $R C L$ circuits, which induce integration, derivations, scaling, and addition operations [7]. In this way, it is highly desirable to dispose of simple linear control-design methodologies with guaranteed stability for the Chua's circuits. In principle, such control methodologies could be extended for a wide variety of industrial systems sharing dynamical and structural characteristics with Chua's circuit [1]. Toward this endeavor, some steps have been taken. In [8], a linear controller to stabilize the Chua's circuit about an equilibrium point is proposed. Given a reference position, say $x_{\text {ref }}$, the controller is able to provide convergence to an equilibrium point $x_{e q}$, which is as close as desired to $x_{\mathrm{ref}}$. Although the effects of uncertain circuit parameters were not studied, the control-design procedure provide important insights on the con-

Manuscript received February 20, 2002; revised June 23, 2002. This paper was recommended by Associate Editor L. Kocarev.

H. Puebla is with Sección de Estudios de Posgrado e Investigación, Escuela Superior de Ingeniería Mecánica y Eléctrica Culhuacan, México D.F. 04430 México, and also with the Programa de Matematicas Aplicadas y Computacion, Instituto Mexicano del Petroleo, Mexico D.F. 04430, México.

J. Alvarez-Ramirez is with Sección de Estudios de Posgrado e Investigación, Escuela Superior de Ingeniería Mecánica y Eléctrica Culhuacan, México D.F. 04430 México, and also with the Programa de Matematicas Aplicadas y Computacion, Instituto Mexicano del Petroleo, Mexico D.F. 04430, México, and the Universidad Autonoma Metropolitana-Iztapalapa, México.

I. Cervantes is with the Sección de Estudios de Posgrado e Investigación, Escuela Superior de Ingeniería Mecánica y Eléctrica Culhuacan, México D.F 04430, México (e-mail: ilse@calmecac.esimecu.ipn.mx).

Digital Object Identifier 10.1109/TCSI.2002.808220 struction of simple linear controllers to regulate the operation of nonlinear circuits. In [9], integral actions are introduced into the control loop to enhance the control performance and achieve exact stabilization about the specified position $x_{\text {ref }}$. Although the authors claim that a rigorous stability proof is provided, unfortunately the analysis is plagued with serious mistakes. For instance, in the analysis of the underlying closed-loop system [9, eq. (A.3) ], the authors miss the effects of interconnection dynamics induced by imperfect knowledge of system vector fields function $f(x)$ in [9, eq. (A.1) ]. In spite of this, the results show that it is necessary to introduce a type of adaptation and/or robustification (e.g., integral actions) scheme in order to cope with functional and parametric circuit uncertainties. Along this line, this brief addresses the problem of designing a tracking controller for nonlinear Chua's circuits involving plant uncertainties and external disturbances. Given a bounded desired trajectory, a tracking controller based on modeling-error compensation is developed. The controller developed here is linear and equivalent to a traditional PI compensator. A rigorous stability analysis of the controlled Chua's circuit is given, which shows that all the states and signals of the closed-loop system are bounded and the tracking error can be made as small as desired by adjusting a tuning parameter. Some extensions of the proposed tacking control scheme to a class of nonlinear circuits is discussed.

\section{PRoblem StATEMENT}

Notation: Throughout this brief, $0_{n}$ denotes the origin of $\mathbb{R}^{n}$ and $x^{T}$ is the transpose of $x$.

Chua's circuit is a simple electronic system that consists of one inductor $L$, two capacitors $C_{1}, C_{2}$, a linear resistor $R$, and a nonlinear resistor $g$. The undriven dynamic equations of Chua's circuit is described by [10]

$$
\begin{aligned}
& \dot{v}_{1}=C_{1}^{-1}\left[R^{-1}\left(v_{1}-v_{2}\right)-g\left(v_{1}\right)\right] \\
& \dot{v}_{2}=C_{2}^{-1}\left[R^{-1}\left(v_{1}-v_{2}\right)+i_{L}\right] \\
& \dot{i}_{L}=-L^{-1} v_{2}
\end{aligned}
$$

where $v_{1}$ and $v_{2}$, are, respectively, the voltages across the capacitors $C_{1}$, and $C_{2}, i_{L}$ is the current through the inductor $L$, and $g\left(v_{1}\right)$ is the current through the nonlinear resistor. It is well known that, for a certain set of circuit parameters, Chua's circuit exhibits periodic and even chaotic behavior [10].

In practical situations, it is commonly desired to induce a prescribed circuit behavior, including fixed-point operation and periodic oscillations. Since the circuit can be unstable around the desired behavior, a feedback controller can be used to stabilize its dynamic behavior. To this end, a control action $u$ represented by an added current at a given circuit node is introduced. The location of the control action $u$ strongly determines the structure of the underlying controller. In this way, as a preliminary step to describe the control problem, certain structural properties of Chua's circuit (1) will be described below. If $q=\left[v_{2}, i_{L}\right]^{T}$, Chua's circuit model (1) can be described as the interconnection of a linear $q$-subsystem and a nonlinear $v_{1}$-subsystem. That is

$$
\begin{aligned}
\dot{v}_{1} & =C_{1}^{-1}\left[R^{-1} v_{1}-g\left(v_{1}\right)\right]+d_{1}^{T} q \\
\dot{q} & =A_{q} q+d_{2} v_{1}
\end{aligned}
$$

where $d_{1}=\left[-C_{1}^{-1} R^{-1}, 0\right]^{T}, d_{2}=\left[C_{2}^{-1} R^{-1}, 0\right]^{T}$, and

$$
A_{q}=\left[\begin{array}{cc}
-C_{2}^{-1} R^{-1} & C_{2}^{-1} \\
-L^{-1} & 0
\end{array}\right] \text {. }
$$


The following result will be useful to establish the location of the control action $u$ in the Chua's circuit.

Lemma 1: Let $v_{1}^{*}(t)$ be an uniformly bounded $v_{1}$ trajectory [i.e., there exists a positive constant $\varsigma$ such that $\left|v_{1}^{*}(t)\right| \leq \varsigma$, for all $t \geq$ 0 ]. Then: a) the $q$ trajectory [corresponding to the dynamic subsystem $\left.\dot{q}(t)=A_{q} q(t)+d_{2} v_{1}^{*}(t)\right]$ is uniformly bounded; b) if in addition $v_{1}^{*}(t)$ is also stationary (e.g., fixed point, periodic orbit, etc.), $q(t)$ converges globally to a unique stationary orbit $q^{*}(t)$.

Proof: a) Since $\operatorname{Trace}\left(A_{q}\right)=-C_{2}^{-1} R^{-1}<0$ and $\operatorname{Det}\left(A_{q}\right)=$ $C_{2}^{-1} L^{-1}>0, A_{q}$ is a (stability) Hurwitz matrix (i.e., all the eigenvalues of $A_{q}$ are in the open left-half complex plane). This implies that the system $\dot{q}(t)=A_{q} q(t)+d_{2} v_{1}^{*}(t)$ is $\mathcal{L}_{2}$ stable (see [11, p. 75]). Consequently, if the input $v_{1}^{*}(t)$ is uniformly bounded, the system trajectory $q(t)$ is also uniformly bounded, for all initial condition $q(0)$. Item b) follows from item a) and the linearity of the $q$-subsystem.

Essentially, the above result states that if one is able to induce a stationary and uniformly bounded trajectory $v_{1}^{*}(t)$, then, the corresponding $q$ trajectory will converge uniformly (with respect to initial conditions) to a unique stationary and uniformly bounded trajectory $q^{*}(t)$. From the control viewpoint, this implies that a feedback control strategy should focus only on the control of the $v_{1}$ subsystem. This is done by introducing a control action $u$ in the $v_{1}$-subsystem as follows:

$$
\begin{aligned}
\dot{v}_{1} & =C_{1}^{-1}\left[R^{-1} v_{1}-g\left(v_{1}\right)+u\right]+d_{1}^{T} q \\
\dot{q} & =A_{q} q+d_{2} v_{1} .
\end{aligned}
$$

In terms of Chua's circuit topology, this is equivalent to add a current $u$ in the nonlinear resistor which is in-phase with the nonlinear-induced current through the resistor $g$. The control model (4) serves as the starting point for the controller design.

The control problem will be considered under the following assumptions.

- Assumption 1. The system states $v_{1}(t)$ and $q(t)=$ $\left[v_{2}(t), i_{L}(t)\right]^{T}$ are available for measurements.

- Assumption 2. The system parameters $\Pi=\left\{R, C_{1}, C_{2}, L\right\}$ are uncertain. However, an estimate $\bar{\Pi}$ of $\Pi$ is available for control design.

- Assumption 3. The nonlinear resistance $g\left(v_{1}\right)$ is a continuously differentiable function.

It should be stressed that Assumption 3 is taken only for stabilityanalysis purposes. As will be shown by means of numerical simulations, the stability of the controlled Chua's circuit holds even in the case where $g\left(v_{1}\right)$ is taken as the standard nondifferentiable function including the absolute value function [12].

Departing from the control model (4) and the result described in Lemma 1, the control problem can be formulated as follows. Given a desired reference signal $v_{1}^{*}(t)$ which is assumed to be bounded and continuously differentiable, the control objective is to design a robust (against model uncertainties and external disturbances) tracking controller with the following characteristics.

a) The feedback controller must be a linear function, so that it can be implemented with standard circuitry.

b) The resulting closed-loop system guarantees that all the states and signals are bounded and the tracking error should be as small as possible.

It should be emphasized that the desired trajectory $\left(v_{1}^{*}(t), q^{*}(t)\right)$ can be e.g., an equilibrium point or a periodic orbit embedded into a chaotic attractor [10].

\section{Controller Design AND Stability Analysis}

The $v_{1}$ subsystem can be written as

$$
\dot{v}_{1}=m\left(v_{1}, q, u\right)+\bar{C}_{1}^{-1}\left[\bar{R}^{-1} v_{1}+u\right]+\bar{d}_{1}^{T} q
$$

where the modeling-error function $m\left(v_{1}, q, u\right)$ is given by

$$
m\left(v_{1}, q, u\right)=-C_{1}^{-1} g\left(v_{1}\right)+a_{1} v_{1}+a_{2} q+a_{3} u
$$

where $a_{1}=C_{1}^{-1} R^{-1}-\bar{C}_{1}^{-1} \bar{R}^{-1}, a_{2}=d_{1}^{T}-\bar{d}_{1}^{T}$ and $a_{3}=-\bar{C}_{1}^{-1}+$ $C_{1}^{-1}$. Notice that the nonlinearity $g\left(v_{1}\right)$ was included in the modeling error because it can not be used for linear control design. Let $\dot{v}_{1}^{*}(t)$ be the time-derivative of the desired reference signal $v_{1}^{*}(t)$. Consider the inverse-dynamics feedback function $u=\varphi_{i n}\left(v_{1}, q, m, v_{1}^{*}\right)$ given by

$$
\begin{aligned}
\varphi_{i n}\left(v_{1}, q,\right. & \left.m, v_{1}^{*}\right)=-\bar{R}^{-1} v_{1} \\
& +\bar{C}_{1}\left[-m\left(v_{1}, q, u\right)-\bar{d}_{1}^{T} q+\dot{v}_{1}^{*}-\omega_{1}\left(v_{1}-v_{1}^{*}\right)\right]
\end{aligned}
$$

where $\omega_{1}>0$ is a prescribed convergence frequency. Under (7), the controlled Chua's circuit can be written as follows:

$$
\begin{aligned}
& \dot{v}_{1}=\dot{v}_{1}^{*}-\omega_{1}\left(v_{1}-v_{1}^{*}\right) \\
& \dot{q}=A_{q} q+d_{2} v_{1} .
\end{aligned}
$$

If $e_{1}(t)=v_{1}(t)-v_{1}^{*}(t)$ is the tracking error, the $v_{1}$-subsystem is equivalent to $\dot{e}_{1}=-\omega_{1} e_{1}$. That is, $v_{1}(t) \rightarrow v_{1}^{*}(t)$ exponentially with a convergence rate of the order of $\omega_{1}$. From Lemma 1, the convergence of the $q$-trajectory to a unique and uniformly bounded trajectory $q^{*}(t)$ is ensured. That is, $q^{*}(t)$ satisfies the differential equality $\dot{q}^{*}(t)=$ $A_{q} q^{*}(t)+d_{2} v_{1}^{*}(t)$. The feedback function $u=\varphi_{\text {in }}\left(v_{1}, q, m, v_{1}^{*}\right)$ can not be implemented as it stands because the modeling-error function $m\left(v_{1}, q, u\right)$ is not available for feedback. To overcome this problem, the following practical feedback controller is proposed:

$$
u=\varphi_{\text {in }}\left(v_{1}, q, \bar{m}, v_{1}^{*}\right)
$$

where $\bar{m}(t)$ is an estimate of the modeling-error signal $m\left(v_{1}(t), q(t), u(t)\right)$ that is obtained by means of the estimator

$$
\dot{\bar{m}}=\omega_{e}[m-\bar{m}] \text {. }
$$

Since $m(t)=\dot{v}_{1}(t)-\bar{C}_{1}^{-1}\left[\bar{R}^{-1} v_{1}(t)+u(t)\right]-\bar{d}_{1}^{T} q(t)$ [see (5)], then

$$
\dot{m}=\omega_{e}\left[\dot{v}_{1}(t)-\bar{C}_{1}^{-1}\left(\bar{R}^{-1} v_{1}+u\right)-\bar{d}_{1}^{T} q-\bar{m}\right]
$$

where $\omega_{e}>0$ is an estimation frequency. To avoid the usage of a derivator in the right-hand side of above equation, the variable $w=$ $\omega_{e}^{-1} \bar{m}-v_{1}$ is introduced to give

$$
\dot{w}=-\bar{C}_{1}^{-1}\left(\bar{R}^{-1} v_{1}+u\right)-\bar{d}_{1}^{T} q-\bar{m}, \quad w(0)=0
$$

such that the estimated modeling-error signal is given by

$$
\bar{m}=\omega_{e}\left(w+v_{1}\right) .
$$

Summarizing, the candidate feedback controller is given by the approximate inverse-dynamics feedback (9) and the modeling-error signal estimator (10), (11). As required, this feedback controller is a linear function of the measured signals $v_{1}(t)$ and $q(t)$. In fact, (9) and (10) can be combined to give $\dot{w}=-\dot{v}_{1}^{*}+\omega_{1} e_{1}$, so that $\bar{m}(t)=-\omega_{e}\left[v_{1}^{*}(t)+v_{1}^{*}(0)\right]+\omega_{e} v_{1}(t)+\omega_{e} \omega_{1} \int_{0}^{t} e_{1}(\sigma) d \sigma$. In this way, the control input $u$ can be written as

$$
u=L_{F F}\left(q, v_{1}^{*}\right)+K_{P} e_{1}+K_{I} \int_{0}^{t} e_{1} d \sigma
$$


where $L_{F F}\left(q, v_{1}^{*}\right)$ is a linear feedforward linear function given by

$$
L_{F F}\left(q, v_{1}^{*}\right)=-\bar{R}^{-1} v_{1}^{*}+\bar{C}_{1} \omega_{e} v_{1}^{*}(0)+\bar{C}_{1} \dot{v}_{1}^{*}-\bar{C}_{1} \bar{d}_{1}^{T} q .
$$

$K_{P}$ and $K_{I}$ are, respectively, proportional and integral gains given by

$$
\begin{aligned}
K_{P} & =-\bar{C}_{1}\left(\bar{C}_{1}^{-1} \bar{R}+\omega_{e}+\omega_{1}\right) \\
K_{I} & =-\bar{C}_{1} \omega_{e} \omega_{1} .
\end{aligned}
$$

That is, the proposed tracking controller is equivalent to a traditionally linear PI compensator (acting on the regulation error $e_{1}=v_{1}-v_{1}^{*}$ ) endowed with a linear feedforward function $L_{F F}\left(q, v_{1}^{*}\right)$. Notice that if one takes $\bar{d}_{1}=0$, the implementation of the proposed controller is even more simple since measurements of the state $q$ are not longer required. This shows that the proposed tracking controller has a simple structure. The stability of the closed-loop system [item b)] will be addressed in the following part of the brief.

\section{A. Stability Analysis}

Let $e_{m}(t)=m(t)-\bar{m}(t)$ be the estimation error. Then the control input can be written as

$$
u=-\bar{R}^{-1} v_{1}+\bar{C}_{1}\left[e_{m}-m\left(v_{1}, q, u\right)-\bar{d}_{1}^{T} q+\dot{v}_{1}^{*}-\omega_{1} e_{1}\right] .
$$

Let $e_{q}(t)=q(t)-q^{*}(t)$ be the $q$-tracking error, and $e_{t r}=\left[e_{1}, e_{q}\right]^{T}$ be the tracking error vector. By recalling that $\dot{q}^{*}(t)=A_{q} q^{*}(t)+d_{2} v_{1}^{*}(t)$ (Lemma 1), and after some straightforward but tedious algebraic manipulations, the controlled Chua's circuits can be written in $\left(e_{t r}, e_{m}\right)$ coordinates as follows:

$$
\begin{aligned}
& \dot{e}_{t r}=A_{c} e_{t r}+E_{1} e_{m} \\
& \dot{e}_{m}=-\omega_{e} \varkappa e_{m}+\phi\left(e_{t r}, e_{m}, \varrho(t)\right)
\end{aligned}
$$

where $\varkappa=C_{1}^{-1} \bar{C}_{1}, E_{1}=\left[1,0_{2}\right]^{T}, \varrho(t)=\left[v_{1}^{*}(t), \dot{v}_{1}^{*}(t), \ddot{v}_{1}^{*}(t)\right]$

$$
A_{c}=\left[\begin{array}{cc}
-\omega_{1} & 0_{2}^{T} \\
d_{2} & A_{q}
\end{array}\right]
$$

$g^{\prime}=d g / d v_{1}$ and

$$
\begin{aligned}
\phi\left(e_{t r}, e_{m}, \varrho(t)\right) \\
=-C_{1}^{-1}\left[R^{-1}-\bar{R}^{-1}-g^{\prime}\left(e_{1}+v_{1}^{*}(t)\right)\right] \\
\quad \cdot\left[-\omega_{1} e_{1}+e_{m}+\dot{v}_{1}^{*}(t)\right]+\left[d_{1}^{T}-\varkappa \bar{d}_{1}^{T}\right]\left[A_{q} e_{q}+d_{2} e_{1}\right] \\
\quad+[1-\varkappa]\left[\omega_{1} e_{m}-\omega_{1}^{2} e_{1}+\ddot{v}_{1}^{*}(t)\right] .
\end{aligned}
$$

System (14) is written in the so-called robust observer form [13], [14]. This system has the following characteristics.

i) Consider the subsystem $\dot{e}_{t r}=A_{c} e_{t r}$. Since the matrix $A_{c}$ is Hurwitz, one has that $e_{r t}(t) \rightarrow 0$ exponentially. This means that the system $\dot{e}_{t r}=A_{c} e_{t r}$ globally satisfies the uniform Lyapunov property (ULP).

ii) Since $\omega_{e} \varkappa>0$, the differential equation $\dot{e}_{m}=-\omega_{e} \varkappa e_{m}$ is globally exponentially stable.

iii) Since $v_{1}^{*}(t)$ is a smooth and bounded time-function for all $t>0$, the time-derivatives $\dot{v}_{1}(t)$ and $\ddot{v}_{1}(t)$ are bounded for all $t>0$. That is, $\varrho(t) \in D_{\varrho}$ for all $t>0$, where $D_{\varrho}$ is a compact domain.
Based on these facts, [14, Lemma 2.4 (see also Remark 2.3)] can be used to state a semiglobal, practical stability result for the controlled Chua's circuit.

Proposition 1: Consider Chua's circuit (1) under the proposed signal tracker (9)-(11). Then, for each pair of compact sets $\mathcal{K}_{r a} \subset \mathbb{R}^{3} \times \mathbb{R}$ and $\mathcal{K}_{u l} \subset \mathbb{R}^{3} \times \mathbb{R}$ with $\mathcal{K}_{u l} \subset \mathcal{K}_{r a}$, neighborhoods of the origin, there exists a positive constant $\omega_{e}^{\min }$ such that all the solutions of the closed-loop system (14), with initial conditions in $\mathcal{K}_{r a}$, are captured by the set $\mathcal{K}_{u l}$, for all $\omega_{e}>\omega_{e}^{\min }$.

Essentially, Proposition 1 states that, given any compact set $\mathcal{K}_{r a}$ of initial conditions $\left(e_{t r}(0), e_{m}(0)\right)^{T}$ (semiglobal stability), there exist values of the estimation parameter $\omega_{e}>\omega_{e}^{\text {min }}$ which guarantee that the Chua's circuit tracks the desired trajectory $\left(v_{1}^{*}(t), q^{*}(t)\right)^{T}$ with arbitrary accuracy given by the size of the compact set $\mathcal{K}_{u l}$ (practical stability). In this way, $\mathcal{K}_{r a}$ can bee seen as an estimate of the region of attraction of the controlled Chua's circuit and $\mathcal{K}_{u l}$ can be seen as the ultimate (finite-time) attractor of the trajectories starting into the set $\mathcal{K}_{\text {ra }}$. From a practical viewpoint, the fact that $\mathcal{K}_{u l}$ is a set of arbitrary size implies that the tracking error can be made as smaller as desired by taking sufficiently large values of the estimation frequency $\omega_{e}$. In this sense, the proposed controller is able to impose the desired circuit dynamics by removing the underlying chaotic dynamics of Chua's circuit. It should be emphasized that the stability analysis can be extended with slight modifications to include time-varying disturbances in the circuit parameters. This will be illustrated via numerical simulations in a subsequent section of the brief.

Although the control structure given by (9)-(11) is similar to the synchronization control developed for communications systems [15], the main difference relies on the fact that, while for communication systems the signal to be tracked is generated by a replication of Chua's circuit, in the control problem addressed in this brief the tracked signal is generated by any source. As a consequence, an autonomous tracking error system has to be analyzed in the former case and a nonautonomous tracking error system has to be studied in the latter case. In this way, the control problem addressed in this brief is of more general nature than the one addressed in communication systems. Specifically, the stability results described above are more general and contain the stability results for communication systems described in [15].

\section{EXtension to a General Class of THIRD-ORder Circuits}

In preceding sections, we have restricted ourselves to Chua's circuit as a benchmark to study chaos control [2], [3], [5], [6]. However, Chua's circuit is a particular case of relative degree [11] one system belonging to a larger class of autonomous third-order systems [16], [17]. Under slight modifications, the results in this brief can be extended to this class of circuits. Some remarks on the extension of the control-design methodology for more general circuits are discussed below.

a) Relative degree one circuits. Similarly to the Chua's circuit, relative degree one circuits can be described as

$$
\begin{aligned}
\dot{x}_{1} & =f\left(x_{1}, q\right)+g\left(x_{1}, q\right) u \\
\dot{q} & =A_{q} q+d x_{1}
\end{aligned}
$$

where $x_{1} \in \mathbb{R}$ and $q \in \mathbb{R}^{2}$ are the components of the state vector. If: a) $y^{*}(t)$ is a desired trajectory for the state $x_{1}$; b) the nonlinearity $f\left(x_{1}, q\right)$ is not exactly known or is not used for control design; and c) $g\left(x_{1}, q\right)$ is bounded away from zero, and $\bar{g}\left(x_{1}, q\right)$ is an estimate of $g\left(x_{1}, q\right)$, the control-design methodology described for Chua's circuit can be extended along the same lines for system (17). 
b) Higher relative degree. Relative degree two and relative degree three systems are considered here. First, consider the relative degree two case, which can be described as follows:

$$
\begin{aligned}
\dot{x}_{1} & =x_{2} \\
\dot{x}_{2} & =f(x, q)+g(x, q) u \\
\dot{q} & =A_{q} q+d^{T} x
\end{aligned}
$$

where $x=\left(x_{1}, x_{2}\right)^{T}$ and $q \in \mathbb{R}$ are the components of the state vector. Let $y^{*}(t)$ be a prescribed trajectory for the state $x_{1}(t)$. By virtue of the chained structure of the system (18), one has that $x_{1}(t) \rightarrow y^{*}(t)$ implies that $x_{2}(t) \rightarrow d y^{*}(t) / d t$. In this way, it is only necessary to specify a trajectory for the state $x_{1}$ in order to specify a desired trajectory for the partial state vector $\left(x_{1}, x_{2}\right)^{T}$. For simplicity in notation, let $y^{*(k)}(t)=d^{k} y^{*}(t) / d t^{k}$. Introduce the tracking errors $z_{i}=x_{i}-y^{*(i-1)}, i=1,2$, or $z=$ $x-Y^{*}$, where $Y_{1}^{*}=\left(y^{*}, y^{*(1)}\right)$. Then, system (18) can be rewritten as

$$
\begin{aligned}
\dot{z}_{1} & =z_{2} \\
\dot{z}_{2} & =y^{*(2)}+F\left(z, q, Y_{1}^{*}\right)+G\left(z, q, Y_{1}^{*}\right) u \\
\dot{q} & =A_{q} q+d^{T}\left(z+Y_{1}^{*}\right)
\end{aligned}
$$

where $F\left(z, q, Y_{1}^{*}\right)=f\left(z+Y_{1}^{*}, q\right)$ and $G\left(z, q, Y_{1}^{*}\right)=$ $g\left(z+Y_{1}^{*}, q\right)$. Given the system description (19), the control task is to achieve $z(t) \rightarrow 0$. The control-design methodology described for Chua's circuit can be extended to the system (19) in the following form. Assume that: a) the state vector $(x, q)^{T}$ is available from measurements; b) $g(x, q)$ is bounded away from zero; c) circuit functions $f(x, q)$ and $g(x, q)$ are not exactly known, but an estimate $\bar{g}(x, q)$ is available for control design; and d) $A_{q}<0$ (i.e., the internal dynamics are $\mathcal{L}_{2}$-stable). In this case, the modeling-error function can be taken as $m\left(z, q, Y_{1}^{*}, u\right)=F\left(z, q, Y_{1}^{*}\right)+\Delta G\left(z, q, Y_{1}^{*}\right) u$, where $\Delta G\left(z, q, Y_{1}^{*}\right)=G\left(z, q, Y_{1}^{*}\right)-\bar{G}\left(z, q, Y_{1}^{*}\right)$ and $\bar{G}\left(z, q, Y_{1}^{*}\right)=g\left(z+Y_{1}^{*}, q\right)$. Hence, system (19) can be rewritten as

$$
\begin{aligned}
\dot{z}_{1} & =z_{2} \\
\dot{z}_{2} & =y^{*(2)}+m\left(z, q, Y_{1}^{*}, u\right)+\bar{G}\left(z, q, Y_{1}^{*}\right) u \\
\dot{q} & =A_{q} q+d^{T}\left(z+Y_{1}^{*}\right) .
\end{aligned}
$$

The corresponding inverse-dynamics feedback function $u=\varphi_{\text {in }}\left(z, q, m, y^{*}\right)$ given by

$$
\begin{aligned}
\varphi_{\text {in }}\left(z, q, m, y^{*}\right) & =\bar{G}\left(z, q, Y^{*}\right)^{-1} \\
& \cdot\left[-y^{*(2)}-m\left(z, q, Y^{*}, u\right)-k_{1} z_{1}-k_{2} z_{2}\right]
\end{aligned}
$$

where the gains $k_{i}, i=1,2$, are chosen such that the polynomial $s^{2}+k_{2} s+k_{1}=0$ has all its roots in the open left-half complex plane. The feedback function (21) can not be implemented as it stands because the modeling-error function $m\left(z, q, Y_{1}^{*}, u\right)$ is unknown. The practical feedback function is obtained when an estimate $\bar{m}(t)$ of the modeling-error signal $m\left(z(t), q(t), Y_{1}^{*}(t), u(t)\right)$ is used instead

$\varphi_{\text {in }}\left(z, q, \bar{m}, y^{*}\right)=\bar{G}\left(z, q, Y_{1}^{*}\right)^{-1}\left[-y^{*(2)}-\bar{m}-k_{1} z_{1}-k_{2} z_{2}\right]$.

If one notes from (20) that $m\left(z, q, Y^{*}, u\right)=\dot{z}_{2}-$ $y^{*(2)}-\bar{G}\left(z, q, Y_{1}^{*}\right) u$, one can use the estimator $\dot{\bar{m}}=\omega_{e}(m-\bar{m})$ to obtain the dynamical estimator $\dot{\bar{m}}=\omega_{e}\left[\dot{z}_{2}-y^{*(2)}-\bar{G}\left(z, q, Y_{1}^{*}\right) u-\bar{m}\right]$. The variable $w=\omega_{e}^{-1} \bar{m}-z_{2}$ is introduced to obtain the following practical controller:

$$
\begin{aligned}
u & =\bar{G}\left(z, q, Y^{*}\right)^{-1}\left[-y^{*(2)}-\bar{m}-k_{1} z_{1}-k_{2} z_{2}\right] \\
\dot{w} & =-y^{*(2)}-\bar{G}\left(z, q, Y_{1}^{*}\right) u-\bar{m} \\
\bar{m} & =\omega_{e}\left(w+z_{2}\right) .
\end{aligned}
$$

The feedback controller contains an integral action. In fact, one can combine the first and the second equations in (23) to obtain $\dot{w}=-k_{1} z_{1}-k_{2} z_{2}$, which is equivalent to $w(t)=w(0)-\int_{0}^{t}\left[k_{1} z_{1}(\sigma)+k_{2} z_{2}(\sigma)\right] d \sigma$. In this way, the modeling-error estimation scheme introduces an integral action acting on the tracking error signal $k_{1} z_{1}(t)+k_{2} z_{2}(t)$. The extension of the present methodology to the relative degree three case is straightforward. Such systems can be described as follows:

$$
\begin{aligned}
& \dot{x}_{1}=x_{2} \\
& \dot{x}_{2}=x_{3} \\
& \dot{x}_{3}=f(x)+g(x) u .
\end{aligned}
$$

Notice the absence of internal dynamics (i.e., the state $q$ is not present). Equation (24) corresponds to the so-called jerk equation where $\dot{x}_{3}=d^{3} x_{1} / d t^{3}$ corresponds to the time derivative of the acceleration [16]. An example of (18) is with $f(x)=$ $-A x_{3}+x_{2}^{2}-x_{1}$, which exhibits chaotic behavior for $u=0$ and $A$ equal to or slightly greater than 2.017. Under similar assumptions as those for the relative degree two case, one can obtain the following tracking control:

$$
\begin{aligned}
u & =\bar{G}\left(z, Y_{2}^{*}\right)^{-1}\left[-y^{*(3)}-\bar{m}-k_{1} z_{1}-k_{2} z_{2}-k_{3} z_{3}\right] \\
\dot{w} & =-y^{*(3)}-\bar{G}\left(z, Y_{2}^{*}\right) u-\bar{m} \\
\bar{m} & =\omega_{e}\left(w+z_{3}\right)
\end{aligned}
$$

where $Y_{2}^{*}=\left(y^{*}, y^{*(1)}, y^{*(2)}\right)^{T}$, and the gains $k_{i}, i=1,2,3$, are chosen such that the polynomial $s^{3}+k_{3} s^{2}+k_{2} s+k_{1}=0$ is Hurwitz. As in preceding cases, the controller (25) contains an integral action on the tracking error function $k_{1} z_{1}+k_{2} z_{2}+k_{3} z_{3}$; namely, $w(t)=w(0)-\int_{0}^{t}\left[k_{1} z_{1}(\sigma)+k_{2} z_{2}(\sigma)+k_{3} z_{3}(\sigma)\right] d \sigma$. In this sense, the feedback controller (23) can be seen as a generalization of the PI controller developed for the Chua's circuit.

Finally, although the stability analysis of the general case involves more complex algebraic manipulations and more subtle analysis tools, the results are essentially the same as in the Chua's circuit (see Proposition 1) namely, the proposed control scheme is able to track the prescribed trajectory $y^{*}(t)$ as close as desired by adjusting the estimation frequency $\omega_{e}>0$. By doing so, one is merely adjusting the rate at which the modeling-error signal is estimated from available circuit measurements.

\section{Simulation EXAMPLE}

For convenience of simulation, the nominal parameters used for control design are given as $\bar{C}_{1}=1.0, \bar{C}_{2}=0.5, \bar{R}=5.0$ and $\bar{L}=1.0$. As in [3], the actual circuit parameters are given by $C_{1}(t)=$ $\bar{C}_{1}+0.1+0.1 \cos (t / 2), C_{2}(t)=\bar{C}_{2}+0.1, R=\bar{R}+\sin (t / 2)$ and 


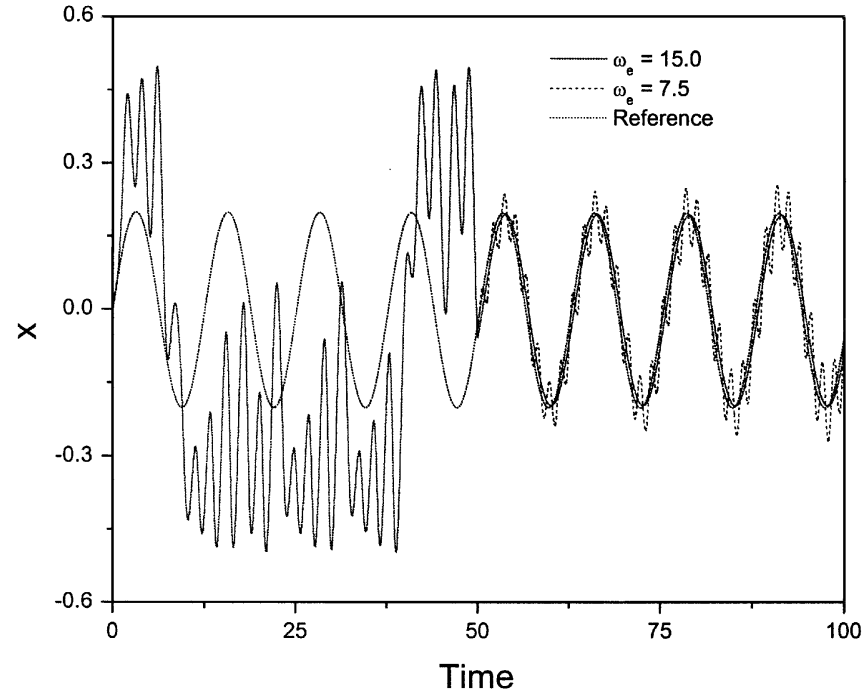

Fig. 1. Performance of the proposed controller for a sinusoidal reference with $\omega_{c}=2.5$ and two different values of the estimation frequency $\omega_{e}$.

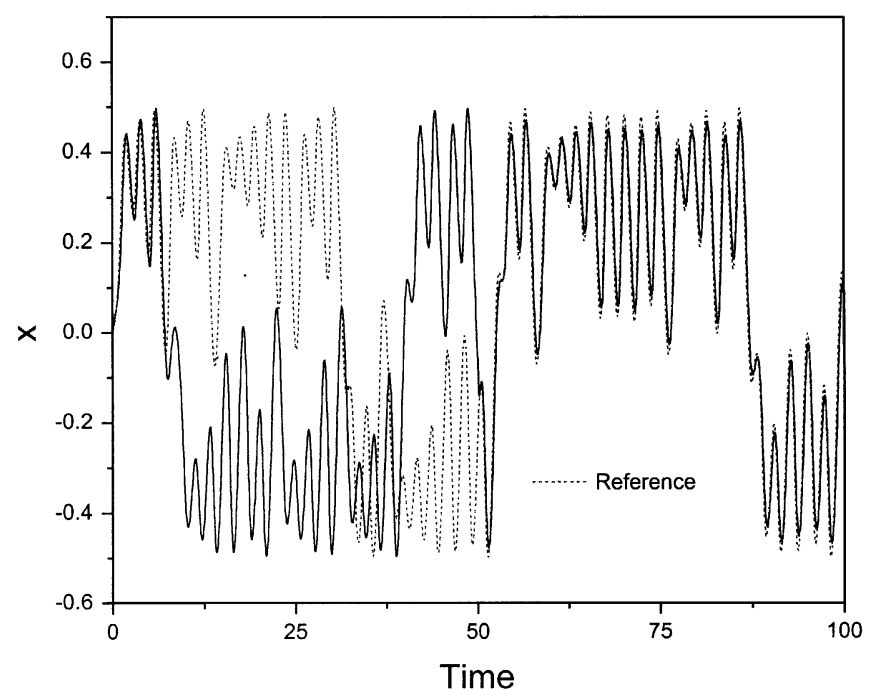

Fig. 2. Performance of the proposed controller for a chaotic reference with $\omega_{c}=7.5$ and $\omega_{e}=12.0$.

$L=\bar{L}+0.1$. For stability analysis convenience, differentiability of the nonlinearity $g\left(v_{1}\right)$ was assumed. Differentiability of $g\left(v_{1}\right)$ is rather a technical requirement of the analysis technique than a necessary stability condition. To illustrate this fact, numerical simulations are carried out by using the standard nondifferentiable function $g\left(v_{1}\right)=R^{-1}\left[m_{0} v_{1}+0.5\left(m_{1}-m_{0}\right)\left\{\left|v_{1}+B_{p}\right|-\left|v_{1}-B_{p}\right|\right\}\right]$, with $m_{0}=-0.37, m_{1}=-0.68$ and $B_{p}=1.08$. Choose the initial conditions $v_{1}(0)=0.01, v_{2}(0)=0.0$ and $i_{L}(0)=0.01$. Let the desired voltage trajectory be either: a) a sinusoidal signal $v_{1}^{*}(t)=0.2 \sin (0.5 t)$ or b) a chaotic reference signal $v_{1}^{*}(t)$ generated from an uncontrolled Chua's circuit with initial conditions $v_{1}(0)=0.1, v_{2}(0)=0.02$ and $i_{L}(0)=0$. The modeling-error compensation approach described before is employed to treat this trajectory planning problems. The simulation results are shown in Fig. 1 for case a) and in Fig. 2 for case b); in both cases the control action starts at $t=50$. As expected from the stability analysis, the size of the tracking error $e_{t r}$ is reduced as the estimation frequency $\omega_{e}$ is increased. These simulation results indicate that the tracking perfor- mance is acceptable and consequently the effects due to parametric uncertainties in Chua's control circuits can be efficiently diminished by the proposed linear control algorithm.

\section{CONCLUSION}

A tracking control design incorporating modeling-error compensation ideas has been proposed for Chua's circuits. Compared with the previous investigations which also address the problem of controlling Chua's circuits, the controller proposed in this brief has a simple linear structure, so that it can be implemented with standard circuitry. A stability analysis is provided to guarantee the stability of the controller, and a simulation example is included to illustrate the performance of the tracking error.

\section{REFERENCES}

[1] G. Chen and X. Dong, From Chaos to Order: Methodologies, Perspectives and Applications. Singapore: World Scientific, 1998.

[2] L. Chen and G. Chen, "Fuzzy predictive control of uncertain chaotic system using time series," Int. J. Bifurcation Chaos, vol. 9, no. 4, pp. 757-767, 1999.

[3] Y.-Ch. Chang, "A robust tracking control for chaotic Chua's circuits via fuzzy approach," IEEE Trans. Circuits Syst. I, vol. 48, pp. 889-895, July 2001.

[4] M. J. Ogorzalek, "Taming chaos-Part II: Control," IEEE Trans. Circuits Syst. I, vol. 40, pp. 700-706, Oct. 1993.

[5] K. Tanaka, T. Ikeda, and H. O. Wang, "A unified approach to control chaos via an LMI-based fuzzy control system design," IEEE Trans. Circuits Syst. I, vol. 45, pp. 1021-1040, Oct. 1998.

[6] J. Xu, G. Chen, and L. S. Shieh, "Digital redesign for controlling the chaotic Chua's circuit," IEEE Trans. Aerosp. Electron. Syst., vol. 32, pp. $1488-1500$, Oct. 1996.

[7] J. J. D'Azzo and C. H. Houpis, Feedback Control Systems Analysis and Synthesis, 2nd ed. Tokyo, Japan: McGraw Hill, 1966.

[8] L. A. B. Torres and L. A. Aguirre, "An extended chaos control method applied to Chua's circuit," Electron. Lett., vol. 35, pp. 768-770, 1999.

[9] R. Femat, J. Capistran-Tobias, and G. Solis-Perales, "Laplace domain controllers for chaos control," Phys. Lett. A, vol. 252, pp. 27-36, 1999.

[10] L. O. Chua, M. Komuro, and T. Matsumoto, "The double scroll family: I and II," IEEE Trans. Circuits Syst. I, vol. CAS-33, pp. 1072-1118, Nov. 1986.

[11] C. A. Desoer and M. Vidyasagar, Feedback Systems: Input-Output Properties. New York: Academic, 1975.

[12] G.-Q. Zhong, "Implementation of Chua's circuit with a cubic nonlinearity," IEEE Trans. Circuits Syst. I, vol. 41, pp. 934-941, Dec. 1994.

[13] F. Esfandiari and H. K. Khalil, "Output feedback stabilization of fully linearizable systems," Int. J. Control, vol. 56, pp. 1007-1037, 1992.

[14] A. Teel and L. Praly, "Tools for semiglobal stabilization by partial state and output feedback," SIAM J. Control Optim., vol. 33, no. 5, pp. 1443-1448, 1995.

[15] H. Puebla and J. Alvarez-Ramirez, "Stability of inverse-system approaches in coherent chaotic communication," IEEE Trans. Circuits Syst. I, vol. 48, pp. 1413-1423, Dec. 2001.

[16] J. C. Sprott, "A new class of chaotic circuit," Phys. Lett. A, vol. 266, pp. 19-23, 2000.

[17] L. A. B. Torres and L. A. Aguirre, "Inductorless Chua's circuit," Electron. Lett., vol. 36, no. 23, pp. 1915-1916, 2000. 